\title{
Low Complexity Transmit Processing for Multibeam Satellite Systems with Non-Linear Channels
}

\author{
Alberto Mengali, Farbod Kayhan, Bhavani Shankar Mysore R. and Björn Ottersten \\ Interdisciplinary Centre for Security, Reliability and Trust, (SnT), University of Luxembourg \\ Email: \{alberto.mengali, farbod.kayhan, bhavani.shankar, bjorn.ottersten\}@uni.lu
}

\begin{abstract}
Aggressive frequency reuse is being considered for multibeam satellites to achieve higher system capacity using available spectrum. Towards achieving the ideal gains of such a reuse, precoding techniques are being considered to minimize the resulting co-channel interference. Most of the precoding techniques, however, do not explicitly consider the distortions introduced by the high power amplifier (HPA), an integral part of the satellite payload, which is inherently non linear. A power efficient amplification introduces non-linear co-channel distortions at the receiver, including the effects of high peak to average power ratios (PAPR), typical of spectrally efficient modulations. This work provides a novel processing at the Gateway comprising a cascade of linear and non-linear operations (Crest Factor Reduction, and Signal Predistortion) to counter the non-linearities and co-channel interference in multibeam satellite systems. The proposed architecture has a lower number of parameters than existing works, leading to efficient coefficient estimation as well as reduced implementation complexity. These parameters are optimized using the simulated annealing algorithm to enhance the Signal to Interference plus Noise ratio at the receiver and the performance is compared with the state-of-the-art.

Index Terms-Multibeam Systems, Frequency Reuse, Signal predistortion, Clipping, Crest Factor Reduction, Simulated Annealing
\end{abstract}

\section{INTRODUCTION}

In the recent years, there is a paradigm shift in multiple antenna terrestrial communications from single user systems to multiple user communications wherein the spatial dimension has been exploited to serve multiple users. Focussing on the downlink transmission, the key issues involve the design of transceiver strategies under different constraints, scheduling multiple users, implementation feasibility and complexity as well as the acquisition and impact of channel state information amongst others [1]. A rich literature exists in terrestrial communications dealing with these issues. With regards to the transmitter processing, both linear (MMSE, Zero-Forcing and those optimized with regards to power and Quality of Service) and non-linear (Dirty Paper Coding, Tomlinson-Harashima precoding) strategies have been proposed yielding significant improvement in system throughput. Some elements of these designs have also been incorporated in latest standards. A similar trend is evolving in satellite systems, as evidenced with the recent studies on multibeam systems employing precoding and user scheduling [2], [3], [4], [5].

While linear and non-linear transmission strategies have been proposed, the underlying radio propagation channel, justifiably, has been assumed linear. The impact of the system components, like the antenna and the amplification (gain), are typically absorbed in this linear model. However, some key components of the communication chain, like the High Power Amplifier (HPA), actually exhibit non-linear characteristics when operated in the high efficiency region (near saturation) [6]. While power amplification serves to boost signal power, its non-linear characteristic and the natural saturation effect introduce distortion in the signal. The severity of distortion introduced depends on the amplitude distribution of the input signal and the required power efficiency. The receiver thus experiences non-linear distortion as well as multiuser cochannel interference. The precoding techniques developed for the linear channel are unable to mitigate the non-linear distortion, thereby mandating the design of non-linear processing at the transmitter. The non-linear distortions can be reduced by operating the HPA in the quasi-linear region; this warrants backing-off the HPA from the saturation by an amount depending on the peak to average power ratio (PAPR) of the input signal. Such an operation with high back-off (especially for higher modulation) drastically reduces the power efficiency and, hence, the resulting signal output power. A common approach to counteract the distortion effect while maintaining the required level of output power, is to preprocess the signal prior to signal amplification. This technique, referred as signal predistortion (SPD) is usually performed in the baseband and is well studied in literature; polynomial functions [7], [8], [9], [10], [11] look up tables (LUT) [12], [13] or iterative interference cancellation [14] have been used to implement SPD. Very large PAPR values, typical of multicarrier signals, result in signal excursions beyond the amplifier saturation point for a significant time. This effect introduces additional distortion that cannot be well-compensated with predistortion; a classic case involves a hard limiter as amplifier, where the cut-off signals cannot be recovered by SPD. This has motivated the use of PAPR reduction in conjunction with SPD [15], [16], [17]. However, these works only consider the effects of the amplifier and do not consider a multi-user channel.

In [18], a non-linear processing at the multiuser transmitter that considers amplifier effects in addition to the linear multiuser interference is presented. The processing comprises multichannel PAPR reduction and SPD. However, the multistream SPD architecture in [18] employs a large number of parameters 
thereby resulting in a complex identification and implementation. In this paper, we propose a non-linear transmission scheme formulated as a cascade of linear multiuser processing, single stream SPD and PAPR reduction blocks. This architecture employs lower number of coefficients, thereby enabling reduced complexity estimation and implementation. Further, the PAPR reduction block now appears at the end of the compensation algorithm, unlike in [18]. While this interchange may sound trivial, it impacts the optimization framework devised to obtain the parameters of these blocks.

The reminder of the paper is organized as follows: Section II present the system model emphasizing the scenario considered and cascade modelling, Section III details the algorithm for optimizing the different blocks of the cascade, Section IV illustrates the results the proposed model while Section V draws conclusions.

Notation: Lower-case bold symbols, $\boldsymbol{a}$, and upper-case bold symbols, $\boldsymbol{A}$, respectively denote vectors and matrices, $T$ denotes the transposition and $|\cdot|$ represents the modulo operator.

\section{SySTEM MODEL}

\section{A. Scenario Description, Space and Ground Segments}

We consider a generic multibeam GEO broadband satellite offering services to fixed users in the Ka-band. The forward link of the system employs DVB-S2x as the air-interface [19]; a return channel to the satellite gateway $(\mathrm{GW})$ is considered towards enabling bidirectional services. A frequency reuse factor of $\beta$ is used with $M$ co-channel beams leading to a total of $\beta M$ beams. We consider one user per beam served by a single carrier in this study; this can be achieved by resorting to Time Division Multiplexing (TDM) of multiple users ${ }^{1}$. Based on the aforementioned description, it suffices to consider a $M$ co-channel beam system with $M$ users (one per beam). We further assume users only have a single antenna.

The antenna of the multibeam satellite comprises a parabolic reflector and an array of feeds allowing the generation of multiple beams. We focus on a single feed per beam configuration [21], [22] where each feed signal is processed by a separate transponder before transmission. Each transponder is a cascade of Input Multiplexing (IMUX) Filter, Automatic Gain Control (AGC), HPA and Output Multiplexing (OMUX) Filter; typical characteristics of these can be obtained from [19].

The GW processing generates $M$ streams for transmission on the $M$ feeds, one per feed. Typically, these streams are multiplexed in frequency on the feeder link and are translated to the appropriate downlink frequency (same for all streams) on-board the satellite. We assume an ideal feeder link and lossless frequency translation on-board the satellite.

\section{B. Signal Model}

Fig. 1 abstracts the multibeam system under consideration where a different stream is transmitted to each of the $M$ single

\footnotetext{
${ }^{1}$ The same can be extended to multiple users per beam on a single carrier by multiplexing them in a DVB-S2x frame [20]
}

antenna users. Let the signal on $k$ th stream at time instance $n$, be

$$
u_{k}(n)=\sum_{m} d_{k}(m) p_{k}\left(n T_{s}-m T\right), \quad k \in[1, M],
$$

where $\left\{d_{k}(m)\right\}$ are the constellation symbols, $p_{k}(\cdot)$ is the pulse-shaping function, $T$ is the symbol duration and $T_{S}$ is the sample duration with $T_{s}=\lambda T$ and $\lambda>1$ is the oversampling factor. The signals $\left\{u_{k}(n)\right\}_{k=1}^{M}$ are input to a SPD

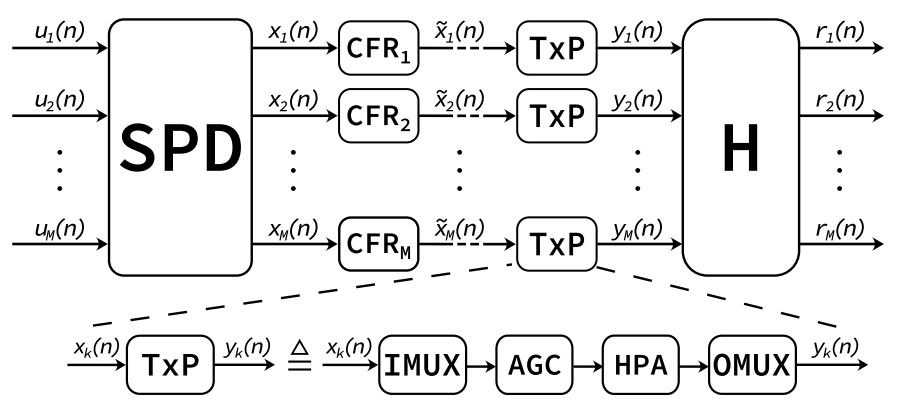

Fig. 1. System Model

block, which processes them jointly to generate $M$ streams, $\left\{x_{k}(n)\right\}_{k=1}^{m}$. These are then passed through a crest factor reduction block aimed at reducing the peak-to-average power ratio (PAPR) of each stream. This is done to limit the distortion introduced by the satellite transponder amplifier non-linear nature. Subsequently, the streams are frequency multiplexed and transmitted by the GW.

The assumptions of lossless feeder links and on-board frequency translations allow us to consider each stream as being processed by a separate transponder section (TxP). The processing involves an IMUX filter to reject out-of-band noise, an AGC that adjusts the signal power according to the chosen Input Backoff (IBO) level, a memoryless HPA and an OMUX filter rejecting out-of-band emissions. Further, the IMUX/ OMUX filters and HPA need not be identical across the TxPs.

The output signal, $\left\{y_{k}(n)\right\}_{k=1}^{M}$ is then radiated on feed $k$ at instance $n$. These co-frequency signals undergo mutual interference over the transmission path from the $M$ transmit antennas to the $M$ receivers; the resulting channel is assumed to be frequency flat and is denoted by a $M \times M$ matrix $\boldsymbol{H}$ [20], [5]. Let $r_{k}(n)$ denote the received signal at user $k$ at instance $n$ and $\boldsymbol{r}(n)=\left[r_{1}(n), r_{2}(n), \ldots, r_{M}(n)\right]^{T}, \boldsymbol{y}(n)=\left[y_{1}(n), y_{2}(n)\right.$, $\left.\ldots, y_{M}(n)\right]^{T}$. With $\boldsymbol{\eta}(n)$ denoting the stacked receiver frontend noise, we have,

$$
\boldsymbol{r}(n)=\boldsymbol{H} \boldsymbol{y}(n)+\boldsymbol{\eta}(n) .
$$

\section{Multistream SPD Model}

A detailed model of the signal predistortion block is depicted in Fig. 2. As can be seen, the predistortion block is internally composed of elementary blocks, each addressed at specific channel impairments. The first of the blocks $(\boldsymbol{C})$ is a linear combiner matrix that mixes the input streams. This is similar to the precoding matrix in standard linear precoding 


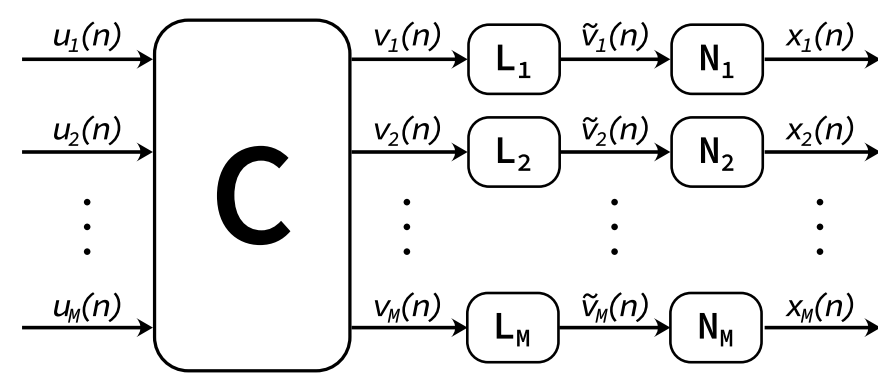

Fig. 2. Predistorter Model

schemes. Unlike [18] where the multi-beam nature of the system was inherent to the whole predistorter block, here it is only explicitly addressed in this linear combiner block. The blocks $L_{k}$ are linear filters and are used to counteract the memory mainly caused by the on-board filters . Further, if these effects can be approximated as being similar to all the streams, an additional estimation complexity reduction can be introduced by making all the filters identical. The blocks $N_{k}$ counteract the non-linear nature of the satellite transponder and are assumed to be memoryless, operating on a sample by sample basis. The combination of a linear dynamic element with a non-linear static one is usually referred in identification theory as a Wiener Model [23]. This model has been shown to be capable of approximating almost any nonlinear system with arbitrarly high accuracy [24], and hence is widely used despite its simple form. By choosing this kind of formulation as opposed to the standard Volterra [11] one as done in [18], we are reducing the the number of coefficients as a function of both the memory and non-linear degree of the predistortion. Doing so effectively limits the overall system complexity, both from the implementation and estimation point of view. The static linear combination block $(\boldsymbol{C})$ can be expressed as a $M \times M$ matrix whose column vectors $\boldsymbol{c}_{k}=\left[c_{1, k}, \cdots, c_{M, k}\right]^{T}, k \in[1, M]$ represent the coefficients of the input-output relation relative to the $k$ th output stream. Such relation can be expressed as

$$
v_{k}(n)=\sum_{l=1}^{M} c_{l, k} u_{l}(n)
$$

Each predistortion block is identified by a memory of $K_{p}$ and a non-linear degree $(2 D-1)$. Towards describing the signal model of the predistorter, we let $\boldsymbol{v}_{k}(n)=\left[v_{k}(n), v_{k}(n+\right.$ $\left.1), \ldots, v_{k}\left(n+K_{p}\right)\right]^{T}, \tilde{v}_{k}(n)$, and $\boldsymbol{a}_{k}=\left[a_{1, k}, \cdots, a_{K_{p}, k}\right]^{T}$ be respectively the input, output and coefficients relative to the linear filter $L_{k}$. In a similar way, we can define as $x_{k}(n)$ and $\boldsymbol{b}_{\boldsymbol{k}}=\left[b_{k, 1}, \cdots, b_{k, D}\right]^{T}$ the output and coefficients of the nonlinear block $N_{k}$. We can now express the input output relation of each block through the following equations:

$$
\begin{aligned}
& \tilde{v}_{k}(n)=v_{k}(n)+\sum_{m=1}^{K_{p}} a_{k, m} v_{k}(n-m), \\
& x_{k}(n)=\sum_{d=1}^{D} b_{k, d} \tilde{v}_{k}(n)\left|\tilde{v}_{k}(n)\right|^{2(d-1)} .
\end{aligned}
$$

By denoting the set of coefficients needed for the predistortion of each stream as $\boldsymbol{w}_{k}=\left[\boldsymbol{c}_{k}^{T}, \boldsymbol{a}_{k}^{T}, \boldsymbol{b}_{k}^{T}\right]^{T}$, we can express the complete set for different streams in compact matrix form as $\boldsymbol{W}=\left[\boldsymbol{w}_{1}, \cdots, \boldsymbol{w}_{M}\right]$. For each stream, the number of coefficients depends both on the number of streams and on the memory and degree of the predistorter model. Referring to Eqs. (2) to (4), we can enumerate the total number of predistorter coefficients as $N_{w}=M\left(M+K_{p}+D\right)$. Where we assumed, without loss of generality, the number of coefficients to be the same for all $k$. By exploiting the channel state information and fixing the combination block $\boldsymbol{C}$ (as the pseudo inverse of the channel for example), the number of parameters to be optimized depends only linearly from the number of streams, further reducing the overall complexity

\section{Crest Factor Reduction Model}

In line with [18], a multistream CFR is described below, where the output of the $k$ th CFR, $\tilde{x}_{k}(n)$, is related to its input, $x_{k}(n)$, as,

$$
\tilde{x}_{k}(n)= \begin{cases}x_{k}(n) & \text { if }\left|x_{k}(n)\right| \leq\left|\gamma_{k}\right|^{2}, \\ \left|\gamma_{k}\right|^{2} x_{k}(n) /\left|x_{k}(n)\right| & \text { if }\left|x_{k}(n)\right|>\left|\gamma_{k}\right|^{2}\end{cases}
$$

where $\gamma_{k}$ is the clipping parameter for the $k$ th stream. Since a joint design of $\left\{\gamma_{k}\right\}$ is employed, we will use $\gamma=$ $\left[\gamma_{1}, \gamma_{2}, \ldots, \gamma_{M}\right]^{T}$. It is worth noting that compared to [18], the CFR block is now placed after the predistortion block rather than before. This choice has been made to allow a finer sensitivity of the CFR block. It is in fact straightforward that the output of the predistorter block would have a wider dynamic range, as opposed to the input, due to the non-linear nature of the predistortion operation. This was not done in [18] due to the increased complexity of the mathematical formulation of the gradient descent when positioning the CFR after the SPD.

\section{Joint CFR And Multistream Predistorter DESIGN}

The objective of this study is to determine the multistream SPD coefficients $\boldsymbol{W}$, and CFR thresholds $\boldsymbol{\gamma}$, that maximize the Signal to Interference plus Noise Ratio (SINR) at the receiver. We can express the SINR of the $k$ th stream as:

$$
\operatorname{SINR}_{k}=\frac{\mathrm{E}\left[\left|u_{k}(n)\right|^{2}\right]}{\mathrm{E}\left[\left|r_{k}(n)-u_{k}(n)\right|^{2}\right]} .
$$

The numerator of Eq. (6) represents the average signal power while the denominator takes into account both the total interference and noise on the $k$ th stream. It is worthy to point out that the effect of the satellite output back-off (OBO) is 
implicitly accounted for in Eq. (6), during the computation of the noise power. In the simulations, in fact, the noise is given through the ratio between the amplifier saturation power $C_{s a t}$ and the noise power $N$. Since the output saturation power is a system parameter that does not vary across the simulations, the noise power is uniquely determined when $C_{\text {sat }} / N$ is fixed. The actual signal power coming out of the amplifier, on the other end, is identified by the OBO value which is dependent on both the signal waveform shape and the HPA operating point. Signals with a higher OBO will exhibit a lower overall signal power which, in turn, will cause a lower SINR.

We can now write our optimization function as

$$
\left\{\boldsymbol{W}_{\text {opt }}, \boldsymbol{\gamma}_{\text {opt }}\right\}=\arg \max _{\{\boldsymbol{W}, \boldsymbol{\gamma}\}}\left[\min \left(\mathrm{SINR}_{k}\right)+\sum_{k=1}^{M} \frac{\mathrm{SINR}_{k}}{M}\right],
$$

where the optimal solution is obtained to provide a maximization of the average received SINR while trying to keep the SINR variation among the beams limited to ensure fairness. The optimization of Eq. (7) is performed using Simulated Annealing [25] jointly on $\boldsymbol{W}$ and $\boldsymbol{\gamma}$. At the beginning of the optimization, an initial temperature $T_{j, k}(0), j \in\left[1, N_{w}+\right.$ $1], k \in[1, M]$ is given to each of the coefficients. At each iteration, one of the coefficients is selected and is moved randomly by adding to it a complex Gaussian variable with standard deviation equal to the coefficient's current temperature. The impact of the change introduced is evaluated by simulating the full system and estimating the new performance by applying equation Eq. (7). If the performance is higher, the new coefficient is kept; else the coefficient is still accepted with a probability that depends on its current temperature. After each iteration, the temperature of all the coefficients is updated using a multiplicative cooling function

$$
T_{j, k}(i+1)=\alpha T_{j, k}(i),
$$

with the cooling coefficient $\alpha<1$ being equal for all $j, k$.

\section{Performance Evaluation}

In this section we numerically evaluate the performance of the proposed multi-stream non-linear processing technique for a selected case of study.

\section{A. Simulation Scenario}

Fig. 3 illustrates the considered scenario having $M=7$ cochannel beams with identical coverage radii of $250 \mathrm{~km}$ and a full frequency reuse $(\beta=1)$. The layout corresponds to the first tier of beams in a classical circular tessellation. We further consider users at the centre of their respective beams in this first study.

Following the works of [3] and [22], we obtain $\boldsymbol{H}=\boldsymbol{\Theta} \boldsymbol{B}$, where $\boldsymbol{B}$ denotes the beam gain matrix and the signal phase due to different propagation paths between the satellite and the users is captured by the diagonal matrix $\boldsymbol{\Theta}$. Given the $i$ th user position, we define $\theta_{k, i}$ as the off-axis angle of the user $i$ with respect to the bore-sight of the beam $k$ and the $3 \mathrm{~dB}$ angle for the $k$ th beam by $\theta_{k 3 \mathrm{~dB}}$. Then the beam gain from $k$ th antenna feed to $i$ th user (the $(i, k)$ element of $\boldsymbol{B}$ ) is approximated as

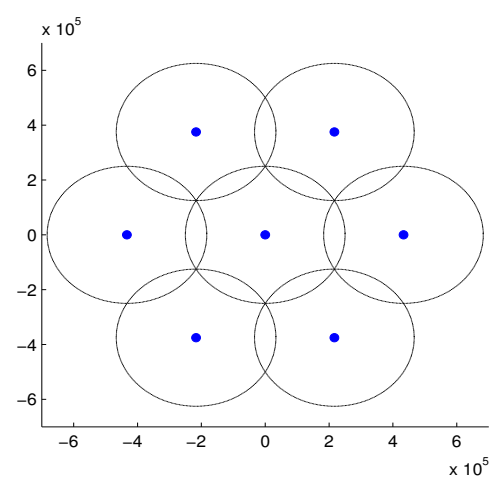

Fig. 3. 7 beam layout considered for simulations.

the square root of $G_{s, k}\left(\frac{J_{1}(u)}{2 u}+36 \frac{J_{3}(u)}{u^{3}}\right)^{2}$ [22]. Here, $G_{s, k}$ is the satellite transmit antenna gain for the $k$ th beam and $u=$ $2.07123 \frac{\sin \left(\theta_{k, i}\right)}{\sin \left(\theta_{k 3 \mathrm{~dB}}\right)}$. Further, $J_{1}$ and $J_{3}$, respectively, are the first and third order Bessel functions of first kind. In this work, we assume $G_{s, k}=1, \theta_{k 3 \mathrm{~dB}}=0.4^{\circ} \quad \forall k$ and $\boldsymbol{\Theta}=\boldsymbol{I}$ [22]

All the streams $\left\{u_{k}(n)\right\}$ employ identical modulations (32 or 64 APSK), symbol rates $R_{S}=4 \mathrm{MBaud}$ and roll-off factors $\rho=0.2$.

Typical responses for the IMUX and OMUX filter are extracted from [19] and appropriately scaled in frequency. Further, we model the HPA using the standard memoryless Saleh model with AM/AM and AM/PM functions taking the form $A(r)=\frac{2 r}{1+r^{2}} ; \Phi(r)=\frac{\pi}{6} \frac{r^{2}}{1+r^{2}}$, respectively [6].

\section{B. Simulation Set-Up}

We first choose the SPD and channel model parameters. In particular, we let, $D=3$ (non-linear model up to the 5-th degree) and $K_{p}=0$ (SPD model is memoryless). While this choice allows for faster simulations, the results obtained are rather conservative due to model mismatches.

As mentioned in Section II-C, we will initialize the linear combination coefficients of the predistorter $\left(c_{l, k}\right)$ with the MMSE precoder resulting from the channel state information, taking the form $\left(\boldsymbol{H}^{*} \boldsymbol{H}+\sigma^{2} \mathbf{I}\right)^{-1} \boldsymbol{H}^{*}$, where $\sigma^{2}$ is the receiver noise variance. By doing so we are fixing the values of the coefficients $c_{l, k}$ which are then not accounted for in the optimization process. The total number of coefficients (SPD $+\mathrm{CFR})$ in the current model is $7(7+3)+7=77$ but due to the constraint put on the linear combinator, the number of coefficients to be estimated is only $7 \cdot 3+7=28$.

The SPD and CFR estimation algorithm, described in Section III, is trained over $10^{6}$ samples corresponding to $10^{5}$ input symbols per stream and an oversampling factor $\lambda=10$. The temperature used in the annealing procedure was dependant on the non-linear degree of the coefficients. The temperatures were 2,0.4 and 0.2 for the coefficients of degree 1,3 and 5 respectively. The temperature update coefficient was set to $\alpha=0.9995$.

For purposes of comparison with the proposed method, we consider, 
- MMSE precoder . This model caters to co-channel interference and ignores the non-linear distortions.

- Joint Predistorter model presented in [18], with a nonlinear degree 3 (the minimum non-linear degree possible considering only odd-polyonmials) and a memory $K_{p}=K_{s}=1$ (This notation corresponds to a memoryless predistortion in [18]). Such a choice of parameters leads to 105 coefficients for optimization, far more than those in the current proposal.

- Single beam using the SPD and CFR system of Fig. 1 with $M=1$. This case does not suffer from co-channel interference and serves as an upper bound.

\section{Performance Evaluation}

We evaluate the resulting Signal to Interference plus Noise Ratio of the central beam (worst performance) and its mean across the remaining beams, subject to lower interference, as a function of the Input Back-off (IBO). We decided to plot the SINR results against IBO instead of OBO to ease the reproducibility of the results. The IBO is in fact a controllable parameter while the value of $\mathrm{OBO}$ is a result of the signal and the components of the satellite transponder. For each scenario, the given results have been obtained by averaging over 30 different realizations of the optimization procedure.

Figs. 4 and 5, respectively, illustrate the central and external beam SINR as a function of IBO for 32 APSK constellations assuming $C_{\text {sat }} / N=20 \mathrm{~dB}$. The achieved SINR values correspond to the region of operation involving 32 APSK [19]. We sweep IBO (Input Back-off) from $2 \mathrm{~dB}$ to $9 \mathrm{~dB}$ in steps of $1 \mathrm{~dB}$ to obtain the different SINR values.

Evidently, the proposed joint multistream CFR and SPD mechanism performs better than MMSE precoder and similarly to the joint design presented in [18] even with the reduced number of coefficients. We can see here that performance is overall better for the central beam while slightly worse on the external ones. This is due to the optimization cost function which in this case, tries to equalize the received SINR across the different beams. This is done to enforce similar performances for different users to achieve a higher fairness. Despite this measure, the central beam user still suffer slightly from the worse channel conditions. Further, the MMSE precoding performs poorly due to the non-linearity and a high IBO is needed to extract meaningful performance from this scheme.

Similar effects are seen for 64 APSK constellations whose performance is plotted in Figs. 6 and 7 for the central and peripheral beams respectively, with a IBO sweeping from 3 to $10 \mathrm{~dB}$. Here the gain of the proposed model with respect to the one in [18] is slightly higher due to the higher non-linear degree of the proposed model. The 64-APSK constellation is characterized by a wider dynamic range leading to a more pronounced distortion on the on-board amplifier. This allows the predistorter model with a higher degree to counteract more effectively the degrading effect of the HPA.

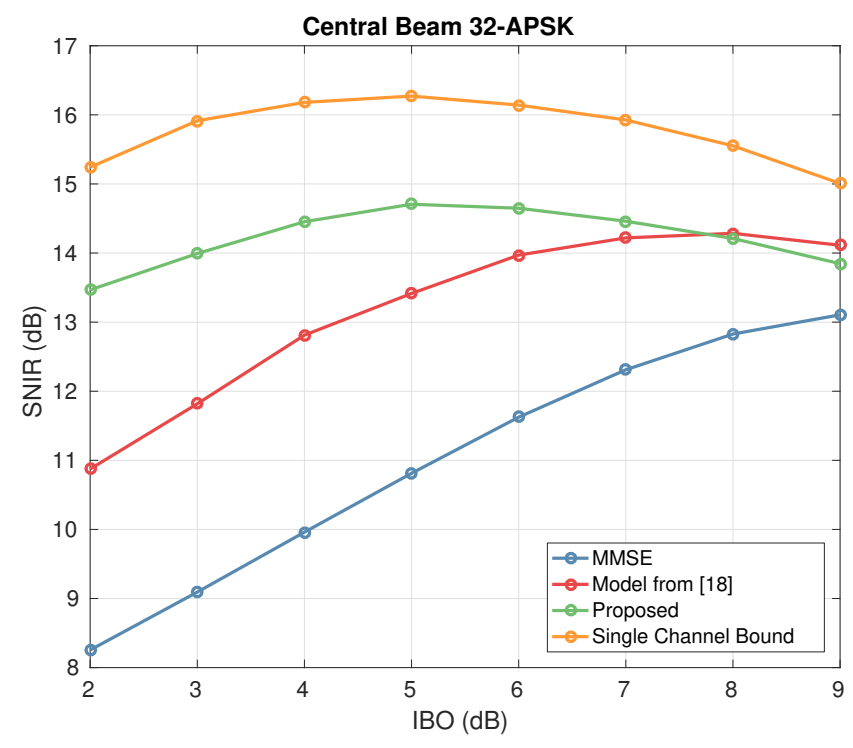

Fig. 4. SINR performance of the central (worst) beam as a function of IBO, $C_{\text {sat }} / N=20 \mathrm{~dB}, 32$ APSK modulation.

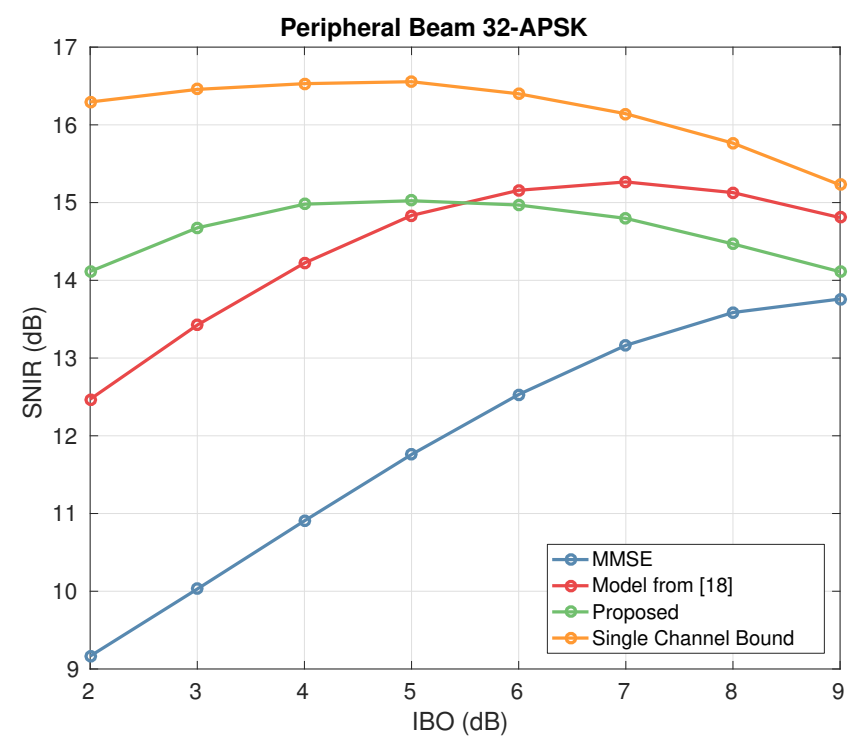

Fig. 5. Average SINR performance of peripheral beams as a function of IBO, $C_{\text {sat }} / N=20 \mathrm{~dB}, 32$ APSK modulation.

\section{CONCLusion}

We propose an update on the GW processing algorithm introudced in [18] for minimizing co-channel interference in multibeam systems when the transponder characteristics are non-linear. This framework comprises multistream SPD and CFR blocks in cascade. The new model exploits a Wiener representation of the SPD block and relies on a separate stream combination block. This allows additional flexibility regarding the optimization of the coefficients while exhibiting lower implementation complexity for higher memory and/ or non linear degree. Simulated Annealing is used to determine the optimal predistortion coefficients and clipping thresholds. The results obtained with the proposed model indicate the attractiveness 


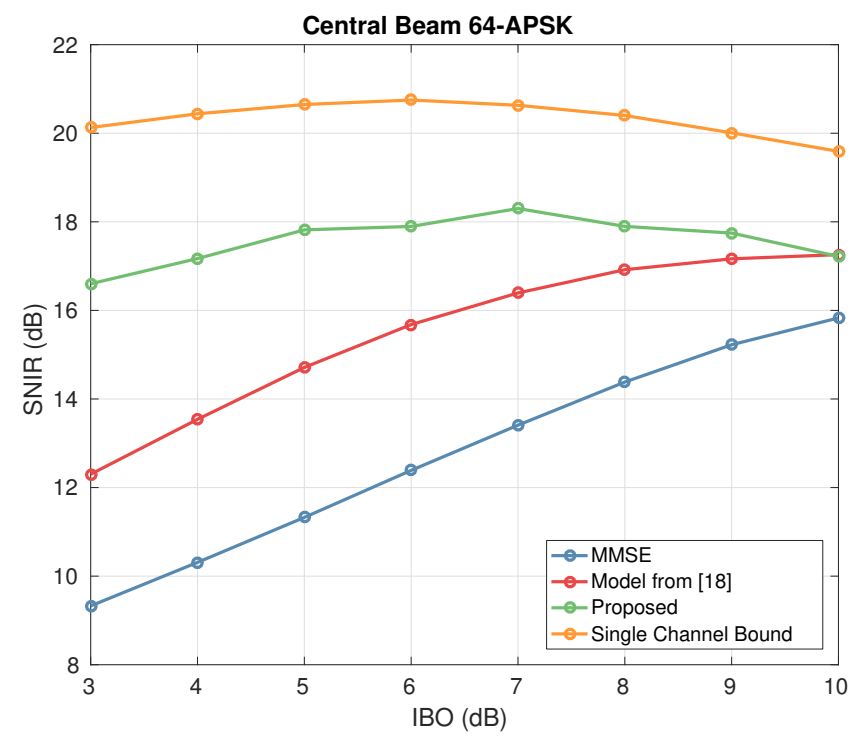

Fig. 6. SINR performance of the central (worst) beam as a function of IBO, $C_{\text {sat }} / N=25 \mathrm{~dB}, 64$ APSK modulation.

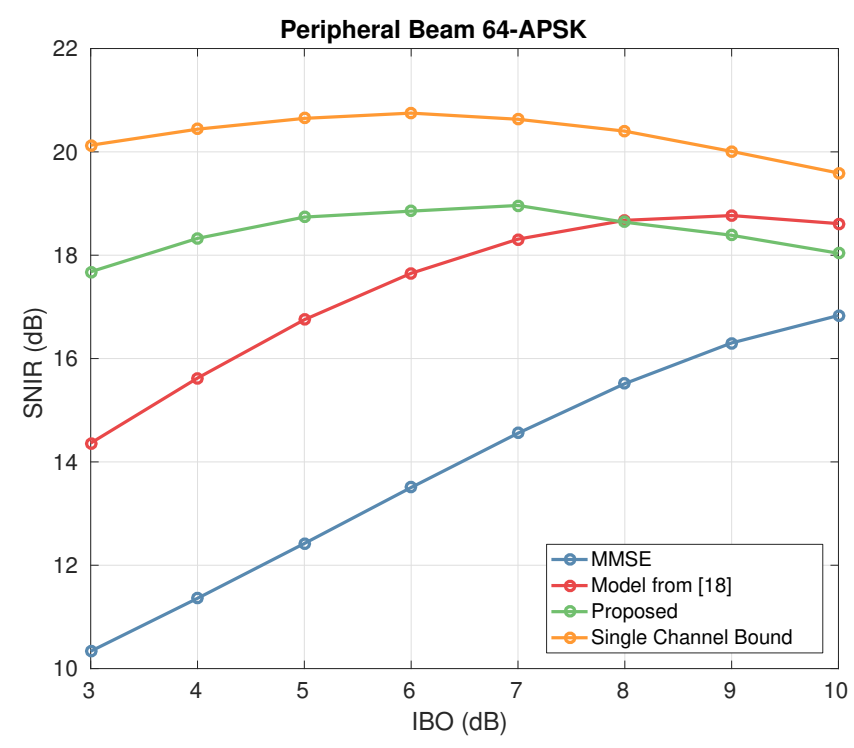

Fig. 7. Average SINR performance of peripheral beams as a function of IBO, $C_{\text {sat }} / N=25 \mathrm{~dB}, 64$ APSK modulation.

of using the proposed reduced complexity method in next generation satellite systems.

\section{ACKNOWLEDGEMENT}

This work was supported by the National Research Fund, Luxembourg under AFR grant for Ph.D. project (Reference 10064089) on Reliable Communication Techniques for Future Generation Satellite Systems.

\section{REFERENCES}

[1] D. Gesbert, M. Kountouris, R. W. Heath, C.-B Chae, and T. Sälzer, "Shifting the MIMO paradigm," IEEE Signal Processing Magazine, vol. 24, no. 5, Sept 2007.
[2] S. Chatzinotas D. Christopoulos and B. Ottersten, "Weighted fair multicast multigroup beamforming under per-antenna power constraints," Signal Processing, IEEE Trans. on, vol. 62, no. 19, pp. $5132-5142$, October 2014.

[3] D. Christopoulos, S. Chatzinotas, and B. Ottersten, "Multicast Multigroup Precoding and User Scheduling for Frame-Based Satellite Communications," IEEE Transactions on Wireless Communications, vol. 14 no. 9, pp. 4695-4707, Sept. 2015.

[4] M. A. Vazquez, Ana Perez-Neira, D. Christopoulos, S. Chatzinotas, B. Ottersten, P.-D Arapoglou, A. Ginesi, and G. Taricco, "Precoding in multibeam satellite communications: Present and future challenges," IEEE Wireless Communication Magazine, Submitted, June 2015.

[5] G. Taricco, "Linear precoding methods for multi-beam broadband satellite systems," in Proc. 20th European Wireless 2014, May 2014

[6] A. A. M. Saleh, "Frequency-independent and frequency-dependent nonlinear models of TWT amplifiers," IEEE Trans. Commun., vol. COM-29, no. 11 , pp. 1715-1720, Nov. 1981.

[7] L. Ding, G. T. Zhou, D. R. Morgan, Z. Ma, J. S. Kenney, J. Kim, and C. R. Giardina, "A robust digital baseband predistorter constructed using memory polynomials," IEEE Trans. Commun., vol. 52, no. 1, pp. 159 - 165, Jan. 2004.

[8] R. Raich, Hua Qian, and G.T. Zhou, "Orthogonal polynomials for power amplifier modeling and predistorter design," IEEE Trans. Veh. Technol., vol. 53, no. 5, pp. 1468 - 1479, Sept. 2004.

[9] D.R. Morgan, Zhengxiang Ma, Jaehyeong Kim, M.G. Zierdt, and J. Pastalan, "A generalized memory polynomial model for digital predistortion of RF power amplifiers," Signal Processing, IEEE Transactions on, vol. 54, no. 10, pp. 3852-3860, Oct 2006.

[10] Lei Ding, Raviv Raich, and G. Tong Zhou, "A Hammerstein predistortion linearization design based on the indirect learning architecture," in Acoustics, Speech, and Signal Processing (ICASSP), 2002 IEEE International Conference on, May 2002, vol. 3, pp. III-2689-III-2692.

[11] C. Eun and E. J. Powers, "A new Volterra predistorter based on the indirect learning architecture," IEEE Trans. on Signal Processing,, vol. 45, no. 1, pp. 223 -227, Jan 1997.

[12] K.J. Muhonen, Mohsen Kavehrad, and R. Krishnamoorthy, "Look-up table techniques for adaptive digital predistortion: a development and comparison," Vehicular Technology, IEEE Transactions on, vol. 49, no. 5, pp. 1995-2002, Sep 2000.

[13] B. Ai, Z.-X. Yang, C.-Y. Pan, S.-G. Tang, and T.-T. Zhang, "Analysis on LUT based predistortion method for HPA with memory," IEEE Trans. on Broadcasting,, vol. 53, no. 1, pp. 127-131, March 2007.

[14] B. F. Beidas, R. I. Seshadri, and N. Becker, "Multicarrier Successive Predistortion for Nonlinear Satellite Systems," IEEE Transactions on Communications, vol. 63, no. 4, pp. 1373-1382, Apr. 2015.

[15] O.A Gouba and Y. Louet, "Joint study of PAPR reduction and digital predistortion," in General Assembly and Scientific Symposium, 2011 XXXth URSI, Aug 2011, pp. 1-4.

[16] C. Nader, P.N. Landin, W. Van Moer, N. Bjorsell, P. Handel, and D. Ronnow, "Peak-power controlling technique for enhancing digital predistortion of RF power amplifiers," Microwave Theory and Techniques, IEEE Transactions on, vol. 60, no. 11, pp. 3571-3581, Nov 2012.

[17] R. Piazza, M. R. Bhavani Shankar, and B. Ottersten, "Generalized direct predistortion with adaptive crest factor reduction control," in Proc. 40th International Conference on Acoustics, Speech and Signal Processing ICASSP, Brisbane, Australia, April 2015.

[18] A. Mengali, B. Shankar, and B. Ottersten, "Joint Predistortion and PAPR Reduction in Multibeam Satellite Systems," To appear in IEEE International Conference on Communications, May 2016, Malaysia, Preprint available online: http://orbilu.uni.lu/handle/10993/25598.

[19] "DVB Document A 171-2: Digital video broadcasting (DVB); implementation guidelines for the second generation framing structure, channel coding and modulation systems for broadcasting, interactive services, news gathering and other broadband satellite applications; Part 2 : DVB-S2 extensions DVB-S2x," March 2015.

[20] D. Christopoulos, P.-D. Arapoglou, S. Chatzinotas, and B. Ottersten, "Linear precoding in multibeam satcoms: Practical constraints," in Proc. 31st AIAA International Communications Satellite Systems Conference (ICSSC), Florence, Oct 2013.

[21] M. Bousquet G. Maral and Z. Sun (Contributing Editor), Satellite Communications Systems: Systems, Techniques and Technology, 5th Edition, Wiley Eastern, 2009.

[22] D. Spano, D. Christopoulos, S. Andrenacci, S. Chatzinotas, B. Ottersten, and J. Krause, "Total degradation analysis of precoded signals onto non- 
linear satellite channels," in Proc. 21st Ka Conference, Bologna, Italy, Oct 2015, [Online]: http://hdl.handle.net/10993/21955.

[23] Fouad Giri, Er-Wei Bai, Manfred Morari, and Manfred Thoma, Eds.

Block-oriented Nonlinear System Identification, vol. 404 of Lecture Notes in Control and Information Sciences, Springer London, London, 2010.

[24] S. Boyd and L. Chua, "Fading memory and the problem of approxi- mating nonlinear operators with volterra series" IEEE Transactions on Circuits and Systems, vol. 32, no. 11, pp. 1150-1161, Nov 1985.

[25] S. Kirkpatrick, C. D. Gelatt, and M. P. Vecchi, "Optimization by Simulated Annealing," Science, vol. 220, no. 4598, pp. 671-680, May 1983. 